\section{GAGA mediates the enhancer blocking activity of the eve promoter in the Drosophila embryo}

\author{
Sumio Ohtsuki and Michael Levine ${ }^{1}$ \\ Department of Molecular and Cellular Biology, Division of \\ Genetics, University of California at Berkeley, Berkeley, \\ California 94720 USA
}

Insulator DNAs and promoter competition regulate enhancer-promoter interactions within complex genetic loci. A transgenic embryo assay was used to obtain evidence that the Drosophila eve promoter possesses an insulator activity that can be uncoupled from the core elements that mediate competition. The eve promoter contains an optimal TATA element and a GAGA sequence. The analysis of various chimeric promoters provides evidence that TATA is essential for promoter competition, whereas GAGA mediates enhancer blocking. The Trithorax-like (Trl) protein interacts with GAGA, and mutations in trl attenuate eve promoter insulator activity. We suggest that Trl-GAGA increases the stability of enhancer-promoter interactions by creating an open chromatin configuration at the core promoter.

Received July 28, 1998; revised version accepted September 9, 1998.

The two major Hox gene clusters in Drosophila, the Antennapedia complex (ANT-C) and the Bithorax complex (BX-C), contain $>100-200 \mathrm{~kb}$ of cis regulatory DNA (e.g., Gorman and Kaufman 1995; Martin et al. 1995). How do the right enhancers interact with the proper promoters? This 'cis trafficking' depends on at least two regulatory mechanisms-insulator DNAs and promoter competition.

Insulators were first identified in the flanking regions of the Drosophila hsp70 locus (Kellum and Schedl 1991, 1992). They are thought to organize hsp 70 within a chromatin loop so that the heat-induced activation of $h s p 70$ does not influence the regulation of neighboring genes and vice versa. Insulators selectively block interactions of distal, not proximal, enhancers with a target promoter (Cai and Levine 1995; Scott and Geyer 1995).

The best characterized insulator is located within the gypsy retrotransposon, which is $340 \mathrm{bp}$ in length and located just downstream of the gypsy 5' LTR. This insulator contains clustered binding sites for the Suppressor of Hairy wing $[\mathrm{Su}(\mathrm{Hw})]$ zinc finger protein, which in turn recruits $\operatorname{Mod}(\operatorname{mdg} 4)$, a protein that suppresses posi-

[Key Words: GAGA; enhancer-promoter interactions; Drosophila embryo; insulators]

${ }^{1}$ Corresponding author.

E-MAIL mlevine@uclink4.berkeley.edu; FAX (510) 643-5785. tion effect variegation (Gerasimova et al. 1995; Gerasimova and Corces 1998). Insulators have been identified within the BX-C (Hagstrom et al. 1996; Zhou et al. 1996; Mihaly et al. 1997), where they have been proposed to organize the extensive cis regulatory DNA into a series of separate chromatin loop domains (e.g., Vasquez et al. 1993).

Promoter competition was first identified in the chicken globin gene cluster (Choi and Engel 1988; Foley and Engel 1992). In principle, a shared enhancer can activate multiple genes but selects the promoter region of just one. Activation of the preferred gene precludes expression of the neighboring genes. Promoter competition has been implicated in the regulation of the Sex combs reduced $(S c r)$ and fushi tarazu $(f t z)$ genes within the ANT-C (Ohtsuki et al. 1998). The $f t z$ autoregulatory enhancer (AE1) is located between the divergently transcribed $S c r$ and $f t z$ genes but selectively interacts with ftz (Pick et al. 1990; Schier and Gehring 1992). This regulatory specificity depends on core promoter elements, particularly TATA. The type $1 \mathrm{ftz}$ promoter contains TATA but lacks the downstream promoter element (Dpe) (Laughon and Scott 1984), whereas type 2 promoters contain initiator (Inr) (Smale 1997) and/or Dpe sequences (Burke and Kadonaga 1996, 1997) but lack TATA (Ohtsuki et al. 1998). Some enhancers, such as AE1, preferentially activate type 1 promoters when given a choice between linked type 1 and type 2 promoters. Others, such as the rhomboid (rho) neuroectoderm enhancer (NEE), promiscuously activate both classes of promoters (Ohtsuki et al. 1998). The regulation of mammalian Hox genes also depends on promoter competition (e.g., Herault et al. 1997; Sharpe et al. 1998).

Here we present evidence that the type 1 even-skipped (eve) promoter possesses an insulator activity, which can be uncoupled from the TATA, Inr, and Dpe core elements. Mutations in a GAGA element, located between TATA and the transcription start site, impair this insulator activity, so that genes residing $5^{\prime}$ of an otherwise normal eve promoter are now activated by a $3^{\prime}$ enhancer. Similar results were obtained in trithorax-like (trl) mutants that diminish the levels of the Trl protein. Mutations in GAGA do not diminish eve promoter function in competition assays. We suggest that Trl-GAGA traps distal enhancers by stabilizing enhancer-promoter interactions.

\section{Results and Discussion}

In the following experiments, white, CAT, and lacZ reporter genes were placed under the control of the type 1 eve promoter and type 2 white promoter, as well as various modified and chimeric promoter sequences. The IAB5 enhancer was used to monitor the activities of these different promoters in transgenic embryos via in situ hybridization. The 1-kb IAB5 enhancer directs expression in the presumptive abdomen of early embryos, and like AE1, it preferentially activates the eve promoter 

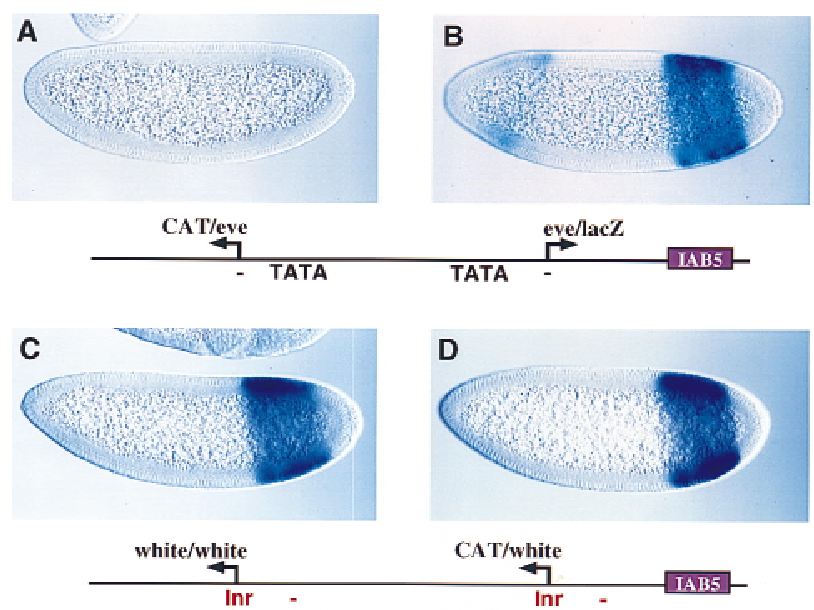

Figure 1. The eve promoter possesses an enhancer blocking activity. Transgenic embryos are undergoing cellularization and are oriented with anterior to the left and dorsal up. Embryos were hybridized with digoxigenin-labeled antisense white, $C A T$, and lacZ RNA probes and stained with anti-digoxigenin antibodies. (A) Embryo contains the eve/CAT-eve/lacZ $\mathrm{P}$-transformation vector indicated in the diagram. It was hybridized with a $C A T$ probe to monitor the expression of the distal eve/CAT reporter gene. Replacing the proximal eve promoter with the white promoter sequence results in the full induction of eve/CAT expression (see Fig. 2A). (B) Same as $A$ except that the embryo was hybridized with a lacZ probe to monitor the expression of the proximal eve/lac $Z$ reporter gene. The weak staining in head regions is due to the P-transformation vector used in these experiments (Small et al. 1992). (C) Embryo contains the white/white-white/CAT P-transformation vector indicated in the diagram. It was hybridized with a white probe to monitor expression of the distal white/white reporter gene. (D) Same as $C$ except that a $C A T$ probe was used to monitor the expression of the proximal white/CAT reporter gene.

when given a choice between eve and white /Ohtsuki et al. 1998).

When the CAT and $1 a c Z$ reporter genes were both placed under the control of the eve promoter (Fig. 1A,B), IAB5 selectively activates the proximal eve/lac $Z$ gene (Fig. 1B) but fails to activate the distal eve/CAT gene (Fig. 1A). In contrast, IAB5 is nearly equally effective at activating both a proximal white/CAT reporter gene (Fig. 1D) and a distal white/white reporter gene (Fig. 1C). These results indicate that the eve promoter, but not white, possesses an enhancer blocking activity.

Various white-eve chimeric promoters were examined to identify the sequences in eve that mediate enhancer blocking. One of these, white eve, contains $5^{\prime}$ sequences (TATA) from eve and 3' sequences (Inr) from white. white $^{\text {eve }}$ contains optimal TATA and Inr elements but is not only virtually inactive (Fig. 2D) but also functions in a dominant-negative fashion to attenuate the activation of a linked eve/CAT gene (Fig. 2C). In this experiment the wild-type eve promoter was placed upstream of a distal CAT reporter gene while the chimeric white eve promoter was attached to the proximal lacZ reporter gene. The residual staining directed by eve/CAT (Fig. $2 \mathrm{C})$ is substantially reduced as compared with control embryos (Fig. 2A). Thus, it would appear that the chi-

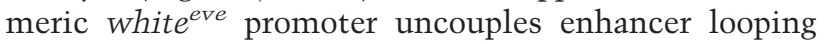
and transcriptional activation; it possesses enhancer blocking activity, even though it is essentially inactive.

The white eve promoter was mutagenized to identify the sequences responsible for enhancer blocking activity. Mutations in the white eve $^{\text {TATA sequence resulted }}$ in only a slight increase in eve/CAT activity (data not shown). The eve and white $e^{e v e}$ promoters contain a
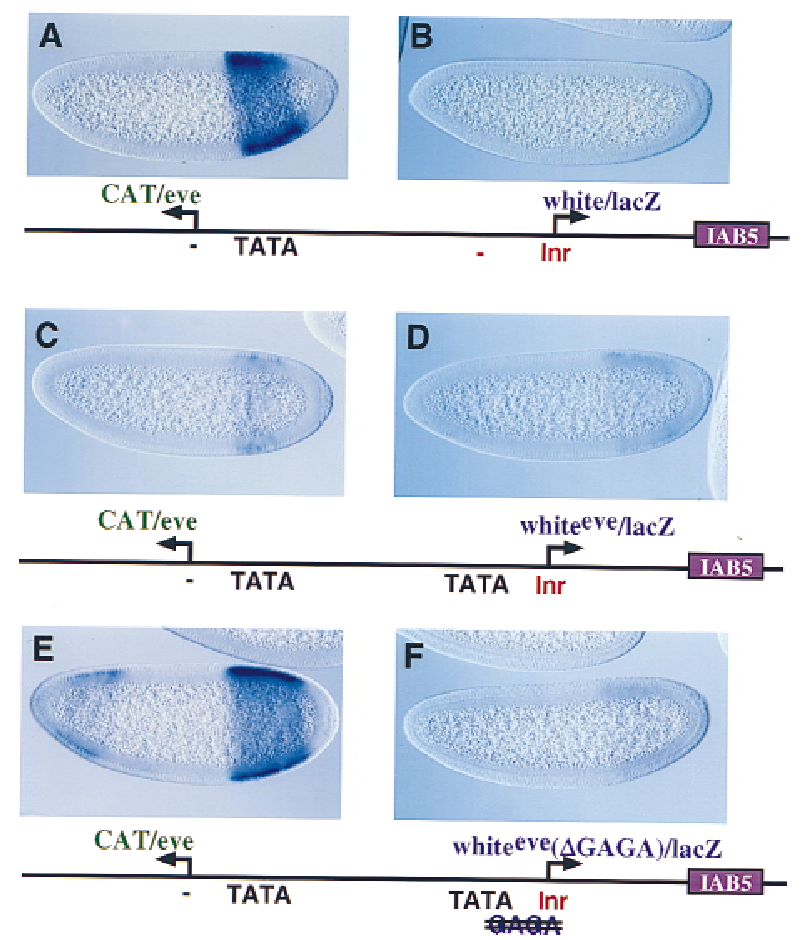

Figure 2. A chimeric eve-white promoter functions in a dominant-negative fashion to block linked genes. Transgenic embryos, oriented with anterior to the left and dorsal up, are at various stages of cellularization and express the transgenes shown in the diagrams beneath the photomicrographs. All embryos shown were stained in parallel, thereby permitting a direct comparison of expression levels. $(A, B)$ eve/CAT-white/ lac $Z$ transgenic embryos with the eve promoter upstream of the distal CAT reporter gene and the white promoter attached to the proximal lacZ gene. The distal $C A T$ gene is fully activated by the 3' IAB5 enhancer $(A)$ and exhibits intense expression in the presumptive abdomen. In contrast, hybridization with a lacZ probe indicates that the proximal white/lac $Z$ gene is inactive. $(C, D)$ Embryos carry a P-transformation vector that is similar to the one shown in $A$ and $B$ except that the proximal white promoter was replaced with the chimeric promoter, white eve. This promoter contains the 5' TATA region from eve and the $3^{\prime}$ Inr region from white, as indicated in the diagram. $(D)$ Hybridization with the lac $Z$ probe reveals that the white ${ }^{\text {eve }}$ promoter is virtually inactive. $(C)$ Hybridization with the CAT probe demonstrates that expression of the distal eve/CAT gene, which contains a completely wild-type eve promoter, is severely attenuated. $(E, F)$ Same as $C$ and $D$ except that the white ${ }^{e v e}$ promoter was mutagenized to disrupt the GAGA element (TATA is intact; see diagram). The modified promoter essentially fails to direct expression of the lacZ reporter $(F)$. However, the distal eve/CAT reporter gene is nearly fully active $(E ; \operatorname{cf} A)$. 
GAGA sequence located between TATA and the transcription start site. A single nucleotide substitution in the white eve GAGA results in nearly normal levels of eve/CAT expression (Fig. 2E, cf. A). These results suggest that GAGA is responsible for the enhancer blocking activity of white $e^{e v e}$, and additional experiments were done to determine whether it has a similar role in the wild-type eve promoter.

Disruption of the eve GAGA element permits the activation of eve/CAT (Fig. 3A,B), without impairing the expression of the mutagenized eve/lacZ gene (Fig. 3B, cf. D). GAGA is bound by the Trl protein, which is maternally expressed and distributed throughout early em-
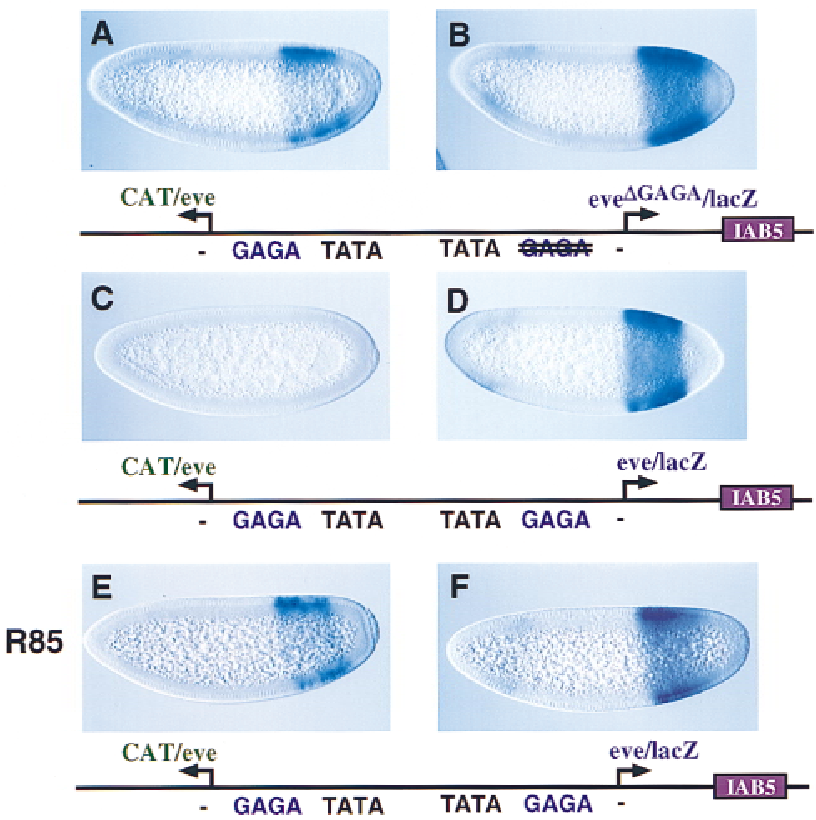

Figure 3. GAGA-Trl interactions mediate the enhancer blocking activity of the eve promoter. Transgenic embryos carry the indicated P-transformation vectors and were hybridized and oriented as described. $(A, B)$ Embryos express $C A T$ and lac $Z$ transgenes that are both driven by the eve promoter, except that the proximal eve/lacZ reporter gene was mutagenized to disrupt the GAGA element (see diagram). The distal eve/CAT gene exhibits moderate levels of expression $(A, \mathrm{cf} . C)$; the proximal eve/lac $Z$ gene is expressed at normal levels $(B, \mathrm{cf} . D)$. It is conceivable that the residual enhancer blocking activity is due to a 'cryptic' GAGA element located in the transcribed region. $(C, D)$ Same as $A$ and $B$ except that both the proximal lacZ and distal $C A T$ genes are attached to normal eve promoter sequences (same as Fig. 1A,B). The proximal, wild-type eve promoter $(D)$ has full enhancer blocking activity so that the distal eve/CAT gene is silent $(C) .(E, F)$ Same as $C$ and $D$ except that the transgene was crossed into an embryo derived from an R85/+ heterozygous female. R85 is a null mutation in the $t r l$ gene, so that these embryos contain half the normal dose of $\mathrm{trl}^{+}$gene activity. This reduction in $\mathrm{Trl}$ attenuates the enhancer blocking activity of the proximal, wild-type eve promoter $(F)$, so that the distal eve/CAT gene is active $(E)$. The levels of eve/CAT expression are similar to those obtained when the proximal GAGA element was mutagenized $(A)$. These results suggest that TrlGAGA interactions are critical for the enhancer blocking activity of the eve promoter. bryos (Farkas et al. 1994; Wilkins and Lis 1997). Mutations in the GAGA element located in the white eve (GAGAG-GAGAT) and eve promoters (GAGAGCACGT) severely reduce Trl binding in gel shift assays (V. Calhoun and M. Levine, unpubl.). Additional evidence for Trl-GAGA interactions stems from gene dosage assays. Females heterozygous for the R85 mutation of $t r l$ were mated with wild-type males carrying the eve/ CAT-eve/lacZ transgene (Fig. 3E,F). Normally, the proximal eve promoter blocks the activation of eve/CAT (Fig. 3C,D). However, the reduction in $\mathrm{trl}^{+}$activity allows the IAB5 enhancer to activate both the proximal eve/lacZ gene (Fig. $3 \mathrm{~F}$ ) and the distal eve/CAT reporter (Fig. 3E).

The IAB5 enhancer preferentially interacts with the TATA-containing eve promoter, even when the TATAless white promoter is inserted between IAB5 and eve (Ohtsuki et al. 1998; see Fig. 2A). Additional assays were conducted to determine whether GAGA participates in this promoter competition process. The IAB5 enhancer was placed $5^{\prime}$ of the divergently transcribed CAT and lacZ reporter genes (Fig. 4). It strongly activates the rightward eve/lacZ gene (Fig. 4B) but only weakly activates white/CAT (Fig. 4A). A mutagenized form of the eve promoter, which lacks the GAGA element, is equally effective in mediating IAB5 activity (Fig. 4D) and attenuating white/CAT expression (Fig. 4C). The white promoter used in these assays is fully active, replacing IAB5 with the promiscuous rho NEE leads to lateral stripes of both white/CAT and eve/lacZ expression (data not shown). Moreover, the white/CAT reporter gene is fully active in the absence of the eve promoter (see Fig. 1D).

These results suggest that GAGA is not essential for eve versus white promoter competition. However, it is conceivable that the white promoter is inherently 'weak;' perhaps GAGA is required for competition between equally 'strong' promoters. This was tested by placing IAB5 5' of eve/CAT and eve/lacZ reporter genes. The genes are expressed at similar levels (Fig. 4E,F), even when the GAGA element is mutagenized in the rightward eve promoter (Fig. 4G,H). Thus, the mutagenized $e v e^{\triangle G A G A}$ promoter is not generally weakened or impaired in promoter competition but is specifically defective in enhancer blocking activity (Fig. 3A,B).

The eve promoter contains TATA and GAGA elements. We have presented evidence that GAGA is essential for enhancer trapping, whereas TATA mediates promoter competition (Ohtsuki et al. 1998). Several observations suggest that these activities can be uncoupled. The $e v e^{\Delta G A G A}$ promoter is impaired in enhancer blocking (Fig. 3) but is just as effective as the wild-type eve promoter in competition assays (Fig. 4). A modified white promoter containing a synthetic TATA element is nearly as active as a linked eve promoter but lacks enhancer trapping activity (Fig. 5A,B; Ohtsuki et al. 1998). This white TATA promoter acquires enhancer blocking activity upon insertion of a GAGA sequence (Fig. 5C,D), as judged by the diminished expression of the distal eve/ CAT gene (Fig. 5C, cf. A). In this experiment, GAGA was 

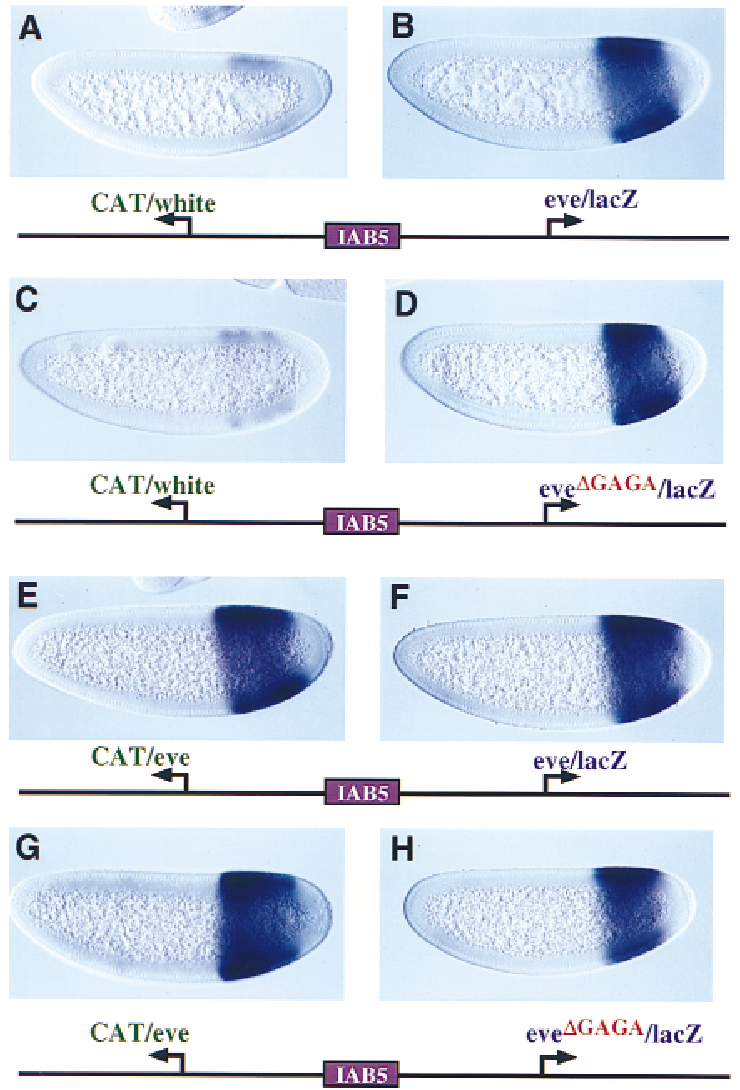

Figure 4. Uncoupling competition and enhancer trapping. The IAB5 enhancer was placed $5^{\prime}$ of the divergently transcribed CAT and $l a c Z$ reporter genes. Transgenic embryos were oriented as described and stained with CAT (left) or lacZ (right) probes. $(A, B)$ The transgene contains the white and eve promoters. As shown previously (Ohtsuki et al. 1998), IAB5 preferentially interacts with the TATA-containing eve promoter $(B)$ and only weakly activates the TATA-less white promoter $(A) .(C, D)$ Same as $A$ and $B$ except that the rightward eve promoter was mutagenized to eliminate the GAGA element. The resulting promoter, $e v e^{\triangle G A G A}$, contains TATA and continues to be the preferred target of the IAB5 enhancer $(D) .(E, F)$ The $C A T$ and lac $Z$ reporter genes are regulated by the wild-type eve promoter. Both reporter genes are strongly activated by IAB5 and exhibit intense staining in the presumptive abdomen. $(G, H)$ Same as $E$ and $F$ except that the rightward eve promoter was mutagenized to eliminate the GAGA element. The resulting eve $e^{\Delta G A G A}$ promoter continues to direct intense expression of lacZ $(H)$ and is essentially as active as the distal eve promoter $(G)$.

inserted between the TATA and Inr elements, and the resulting promoter (white ${ }^{T A T A+G A G A}$ ) functions in a dominant-negative fashion, as seen for white ${ }^{e v e}$ (Fig. 2). However, the insertion of GAGA $5^{\prime}$ of TATA results in

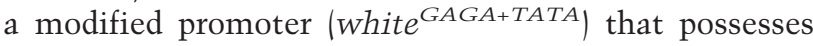
enhancer trapping activity (Fig. 5E, cf. A) but is fully active (Fig. 5F, cf. D). These results suggest that the dominant-negative activities of the white eve and white ${ }^{T A T A+G A G A}$ promoters depend on the positioning of GAGA between optimal TATA and Inr elements.

Promoters that possess enhancer blocking activities should facilitate the orderly trafficking of cis-regulatory elements. For example, eve stripe enhancers located $3^{\prime}$ of the transcription unit should be unable to interact with neighboring genes located $5^{\prime}$ of eve. Similarly, the ftz promoter contains a GAGA element located $5^{\prime}$ of TATA. Based on our analysis of white eve, white TATA+GAGA $^{\text {end }}$ white GAGA+TATA this configuration of core elements should allow the ftz promoter to be both transcriptionally active and able to block distal enhancers. Perhaps the $f t z$ promoter helps inhibit interactions between $3^{\prime}$ Antp enhancers and $5^{\prime}$ homeotic genes [ $D f d$ (Deformed) and $S c r$ ] within the ANT-C. It is conceivable that many promoters possess an intrinsic enhancer blocking activity. Inspection of 250 Drosophila promoter sequences (Arkhipova 1995) reveals that $\sim 15 \%$ contain at least one optimal GAGA element within 50 bp $5^{\prime}$ of the transcrip-
A
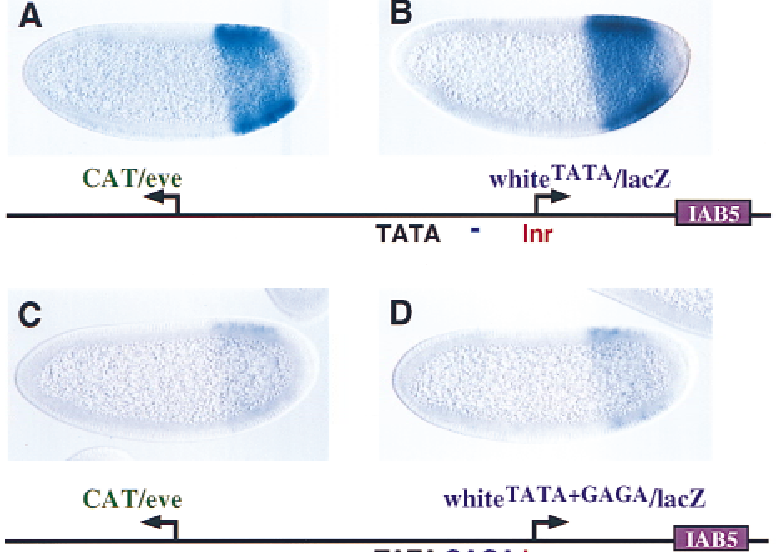

E

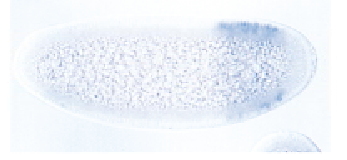

CAT/eve
B

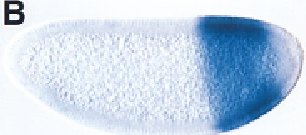

TATA

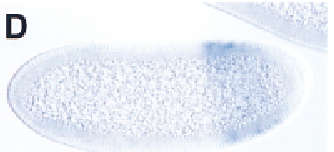

hite TATA+GAGA/lacZ

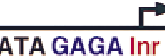

$\mathbf{F}$
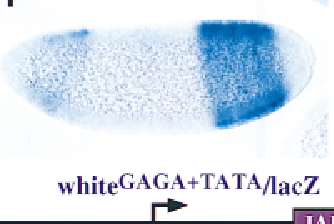

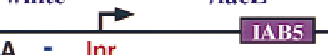

Figure 5. Changing the location of GAGA. Transgenic embryos carry the indicated P-transformation vectors and were hybridized and oriented as described. Embryos were stained in parallel, thereby alowing direct comparison of relative expression levels. $(A, B)$ The distal CAT gene is under the control of the normal eve promoter; the proximal lacZ gene was attached to a modified white promoter containing an optimal TATA element (see Ohtsuki et al. 1998). The 3' IAB5 enhancer interacts with both promoters to direct nearly equal levels of $C A T(A)$ and lacZ $(B)$ expression. The normal white promoter, lacking TATA, is not activated by IAB5 in similar transgenes (e.g., Fig. 2B). $(C, D)$ Same as $A$ and $B$ except that the proximal white ${ }^{T A T A}$ promoter was further modified to include a GAGA element between TATA and the Inr. This promoter exhibits the same type of dominant-negative activity as white eve (see Fig. 2D). (E,F) Same as $C$ and $D$ except that the GAGA element was placed 28 bp $5^{\prime}$ of TATA. The resulting promoter, white GAGA+TATA, traps the IAB5 enhancer so that $C A T$ expression $(E)$ is weak (cf. $A$ ). However, unlike the white $e^{T A T A+G A G A}$ promoter $(D)$, this promoter is fully active $(F)$. 
tion start site. An earlier analysis of one of these promoters, $\alpha 1$-tubulin, indicates that GAGA helps insulate tubulin expression from position effects $\left(\mathrm{O}^{\prime}\right.$ Donnell et al. 1994).

The enhancer blocking activity of the eve promoter appears to be mediated by interactions of $\operatorname{Trl}$ with GAGA. Trl has been shown to recruit the NURF protein complex, which facilitates the binding of upstream activators or core polymerase II components by decondensing chromatin (Tsukiyama and Wu 1997; Wilkins and Lis 1997). Trl-GAGA might trap distal enhancers by increasing the stability of enhancer-promoter interactions through the creation of an open chromatin configuration or by increasing the occupancy of core Pol II components such as TFIID.

\section{Materials and methods}

P-transformation assays and genetic crosses

$y w^{67}$ flies were used for all P-transformation assays. Fusion genes were introduced into the Drosophila germ line as described by Small et al. (1992). Between three and seven independent transformed lines were generated for each construct, and at least three separate lines were examined by in situ hybridization. Embryos were collected, fixed, and hybridized with digoxigenin-labeled white, CAT, and $1 a c Z$ antisense RNA probes exactly as described (Tautz and Pfeifle 1989; Small et al. 1992).

The genetic cross used in the experiment presented in Figure 3 (E,F) was done as follows. Females heterozygous for the R85 trl mutant (Bhat et al. 1996) were mated with $y w^{67}$ transgenic males carrying the eve/ $C A T-e v e /$ lac $Z$ transgene. $\mathrm{F}_{1}$ embryos were collected and fixed, and hybridized as described previously (Tautz and Pfeifle 1989; Jiang et al. 1991). The reciprocal cross, $y w^{67}$ transgenic females mated with $\mathrm{R} 85 /+$ males, does not impair the enhancer blocking activity of the proximal eve promoter (S. Ohtsuki, unpubl.). This observation suggests that maternal Trl products interact with eve.

\section{Preparation of P-transformation vectors}

The mini-white promoter region that was used in this study extends from -316 bp upstream of the transcription start site to $+174 \mathrm{bp}$. The eve promoter extends from $-34 \mathrm{bp}$ to $+166 \mathrm{bp}$. The chimeric white ${ }^{e v e}$ promoter was prepared by fusing 5 ' eve promoter sequences, from -31 to -1 bp, with 3 ' white sequences $(-1$ to $+174 \mathrm{bp})$. This fusion was made by PCR and confirmed by DNA sequence analysis. The mutagenized white $^{\text {eve }}$ promoter lacking TATA was prepared with a mutagenic oligonucleotide that converts the sequence GTATAAAAG into GGAGCAAAG. The mutagenized white $e^{e v e}$ promoter lacking GAGA was prepared with a mutagenic oligonucleotide that converts the sequence TGAGAGCAGTT into TGAGATCAGTT (the +1 site is underlined). This mutation converts the G residue at position 6 of the GAGAG motif into T. Previous studies have shown that this substitution markedly reduces the binding of the Trl protein (Omichinski et al. 1997). The GAGA element was disrupted in the eve promoter with a mutagenic oligonucleotide that converts the sequence TGAGAGCA into TCACTGCA (the +1 site is underlined).

$\bar{T}$ The white/CAT/lacZ P-transformation vector that was used for all the experiments presented in this study is a modification of pCasPer, which contains divergently transcribed white and lacZ reporter genes (Small et al. 1992). It was modified by insertion of a CAT reporter gene between white and lacZ, as described by Ohtsuki et al. (1998).

The eve, white, white eve, and various modified promoters were isolated as $A s c \mathrm{I}-\mathrm{BamHI}$ fragments and cloned into a unique BamHI site located at the $5^{\prime}$ end of either the $C A T$ or $1 a c Z$ coding sequence present in pBluescript vectors. The $C A T$ fusion genes were subsequently isolated as $A s c \mathrm{I}-N o t \mathrm{I}$ fragments and used to replace the AscI-NotI CAT fragment in the pCasPer vector. The lacZ fusion genes were isolated as AscI-XbaI fragments and used to replace the AscI-XbaI lacZ fragment in the pCasPer vector. For most of the experiments, the IAB5 enhancer was isolated as a $1-\mathrm{kb}$ PstI-Pst I fragment and cloned into a unique Pst I site located $3^{\prime}$ of the $1 a c Z$ reporter gene. IAB5 was placed in a $5^{\prime}$ position of the vector (Fig. 4) by isolating it as a 1-kb AscI-AscI fragment and cloning it into the unique $A s c$ I site located between the divergently transcribed $C A T$ and lacZ genes located in the pCasPer vector.

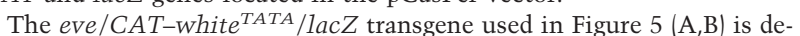
scribed in Ohtsuki et al. (1998). GAGA was inserted either 3' (Fig. 5C,D) or 5' (Fig. 5E,F) of TATA by PCR mutagenesis. The 3' GAGA was created by altering nucleotides between -7 and -3 bp upstream of the transcription start site (CGCCT-GAGAG). The 5' GAGA was made by altering nucleotides between -61 and -57 bp upstream of the start site (CTGCGGAGAG).

\section{Acknowledgments}

We thank Dale Dorsett for helpful discussions and Krishna Bhat for fly stocks. We also thank Vincent Calhoun for analyzing core promoter sequences. This work was funded by a grant from the National Institutes of Health (GM34431). S.O. is a fellow of the Human Frontiers Science Program.

The publication costs of this article were defrayed in part by payment of page charges. This article must therefore be hereby marked 'advertisement' in accordance with 18 USC section 1734 solely to indicate this fact.

\section{References}

Arkhipova, I.R. 1995. Promoter elements in Drosophila melanogaster revealed by sequence analysis. Genetics 139: 1359-1369.

Bhat, K.M., G. Farkas, F. Karch, H. Gyurkovics, J. Gausz, and P. Schedl. 1996. The GAGA factor is required in the early Drosophila embryo not only for transcriptional regulation but also for nuclear division. Development 122: 1113-1124.

Burke, T.W. and J.T. Kadonaga. 1996. Drosophila TFIID binds to a conserved downsteam basal promoter element that is present in many TAT-box-deficient promoters. Genes \& Dev. 10: 711-724.

. 1997. The downstream core promoter element, DPE, is conserved from Drosophila to humans and is recognized by TAFII60 of Drosophila. Genes \& Dev. 11: 3020-3031.

Cai, H. and M. Levine. 1995. Modulation of enhancer-promoter interactions by insulators in the Drosophila embryo. Nature 376: 533-536.

Choi, O.R. and J.D. Engel. 1988. Developmental regulation of beta-globin gene switching. Cell 55: 17-26.

Dorsett, D. 1993. Distance-independent inactivation of an enhancer by the suppressor of Hairy-wing DNA-binding protein of Drosophila. Genetics 134: 1135-1144.

Farkas, G., J. Gausz, M. Galloni, G. Reuter, H. Gyurkovics, and F. Karch. 1994. The Trithorax-like gene encodes the Drosophila GAGA factor. Nature 371: 806-808.

Foley, K.P. and J.D. Engel. 1992. Individual stage selector element mutations lead to reciprocal changes in $\beta$ vs. $\epsilon$-globin gene transcription: Genetic confirmation of promoter competition during globin gene switching. Genes \& Dev. 6: 730-744.

Gerasimova, T.I. and V.G. Corces. 1998. Polycomb and trithorax group proteins mediate the function of a chromatin insulator. Cell 92: 511521.

Gerasimova, T.I., D.A. Gdula, D.V. Gerasimov, O. Simonova, and V.G. Corces. 1995. A Drosophila protein that imparts directionality on a chromatin insulator is an enhancer of position-effect variegation. Cell 82: 587-597.

Geyer, P.K. 1997. The role of insulator elements in defining domains of gene expression. Curr. Opin. Genet. Dev. 7: 242-248.

Geyer, P.K. and V.G. Corces. 1992. DNA position-specific repression of transcription by a Drosophila zinc finger protein. Genes \& Dev. 6: 1865-1873.

Gorman, M.J. and T.C. Kaufman. 1995. Genetic analysis of embryonic cis-acting regulatory elements of the Drosophila homeotic gene sex combs reduced. Genetics 140: 557-572.

Hagstrom, K., M. Muller, and P. Schedl. 1996. Fab-7 functions as a chromatin domain boundary to ensure proper segment specification by the Drosophila bithorax complex. Genes \& Dev. 10: 3202-3215.

Henikoff, S. 1996. Dosage-dependent modification of position-effect variegation in Drosophila. BioEssays 18: 401-409.

Herault, Y., N. Fraudeau, J. Zakany, and D. Duboule. 1997. Ulnaless (U1), a regulatory mutation inducing both loss-of-function and gain-of- 


\section{Ohtsuki and Levine}

function of posterior Hoxd genes. Development 124: 3493-3500.

Jiang, J., D. Kosman, Y.T. Ip, and M. Levine. 1991. The dorsal morphogen gradient regulates the mesoderm determinant twist in early Drosophila embryos. Genes \& Dev. 5: 1881-1891.

Kellum, R. and P. Schedl. 1991. A position-effect assay for boundaries of higher order chromosomal domains. Cell 72: 741-752.

- 1992. A group of scs elements function as domain boundaries in an enhancer-blocking assay. Mol. Cell. Biol. 12: 2424-2431.

Laughon, A. and M.P. Scott. 1984. Sequence of a Drosophila segmentation gene: protein structure homology with DNA-binding proteins. Nature 310: 25-31.

Martin, C.H., C.A. Mayeda, C.A. Davis, C.L. Ericsson, J.D. Knafels, D.R. Mathog, S.E. Celniker, E.B. Lewis, M.J. Palazzolo. 1995. Complete sequence of the bithorax complex of Drosophila. Proc. Natl. Acad. Sci. 92: 8398-8402.

Mihaly, J., J. Hogga, I. Gausz, H. Gyurkovics, and F. Karch. 1997. In situ dissection of the Fab-7 region of the bithorax complex into a chromatin domain boundary and a Polycomb-response element. Development 124: 1809-1820.

O’Donnell, K.H., C.T. Chen, and P.C. Wensink. 1994. Insulating DNA directs ubiquitous transcription of the Drosophila melanogaster alpha 1-tubulin gene. Mol. Cell. Biol. 14: 6398-6408.

Ohtsuki, S., M. Levine, and H.N. Cai. 1998. Different core promoters possess distinct regulatory activities in the Drosophila embryo. Genes \& Dev. 12: 547-556.

Omichinski, J.G., P.V. Pedone, G. Felsenfeld, A.M. Gronenborn, and G.M. Clore. 1997. The solution structure of a specific GAGA factorDNA complex reveals a modular binding mode. Nat. Struct. Biol. 4: 122-132.

Pick, L., A. Schier, M. Affolter, T. Schmidt-Glenewinkel, and W.J. Gehring. 1990. Analysis of the ftz upstream element: Germ layer-specific enhancers are independently autoregulated. Genes \& Dev. 4: 1224-1239.

Schier, A.F. and W.J. Gehring. 1992. Direct homeodomain-DNA interaction in the autoregulation of the fushi tarazu gene. Nature 356: 804 807.

Scott, K.S. and P.K. Geyer. 1995. Effects of the su(Hw) insulator protein on the expression of the divergently transcribed Drosophila yolk protein genes. EMBO J. 14: 6258-6267.

Sharpe, J., S. Nonchev, A. Gould, J. Whiting, and R. Krumlauf. 1998. Selectivity, sharing and competitive interactions in the regulation of Hoxb genes. EMBO J. 17: 1788-1798.

Smale, S.T. 1997. Transcription initiation from TATA-less promoters within eukaryotic protein-coding genes. Biochim. Biophys. Acta. 1351: 73-88.

Small, S., A. Blair, and M. Levine. 1992. Regulation of even-skipped stripe 2 in the Drosophila embryo. EMBO J. 11: 4047-4057.

Tautz, D. and C. Pfeifle. 1989. A non-radioactive in situ hybridization method for the localization of specific RNAs in Drosophila embryos reveals translational control of the segmentation gene hunchback. Chromosoma 98: 81-85.

Tsukiyama, T. and C. Wu. 1997. Chromatin remodeling and transcription. Curr. Opin. Genet. Dev. 7: 182-191.

Vasquez, J., G. Farkas, M. Gaszner, A. Udvardy, H. Muller, K. Hagstrom, H. Gyurkovics, L. Sipos, J. Gausz, M. Galloni, I. Hogga, F. Karch, and P. Schedl. 1993. Genetic and molecular analysis of chromatin domains. Cold Spring Harbor Symp. Quant. Biol. 58: 45-54.

Wilkins, R.C. and J.T. Lis. 1997. Dynamics of potentiation and activation: GAGA factor and its role in heat shock gene regulation. Nucleic Acids Res. 25: 3963-3968.

Zhou, J., S. Barolo, P. Szymanski, and M. Levine. 1996. The Fab-7 element of the bithorax complex attenuates enhancer-promoter interactions in the Drosophila embryo. Genes \& Dev. 10: 3195-3201. 


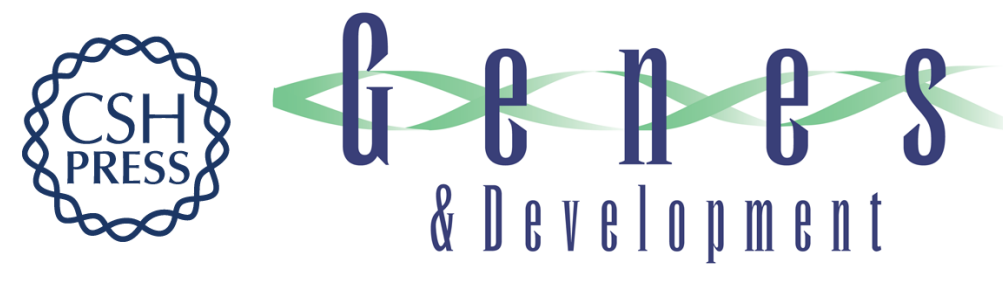

\section{GAGA mediates the enhancer blocking activity of the eve promoter in the Drosophila embryo}

Sumio Ohtsuki and Michael Levine

Genes Dev. 1998, 12:

Access the most recent version at doi:10.1101/gad.12.21.3325

References This article cites 37 articles, 20 of which can be accessed free at: http://genesdev.cshlp.org/content/12/21/3325.full.html\#ref-list-1

License

Email Alerting

Receive free email alerts when new articles cite this article - sign up in the box at the top Service right corner of the article or click here.

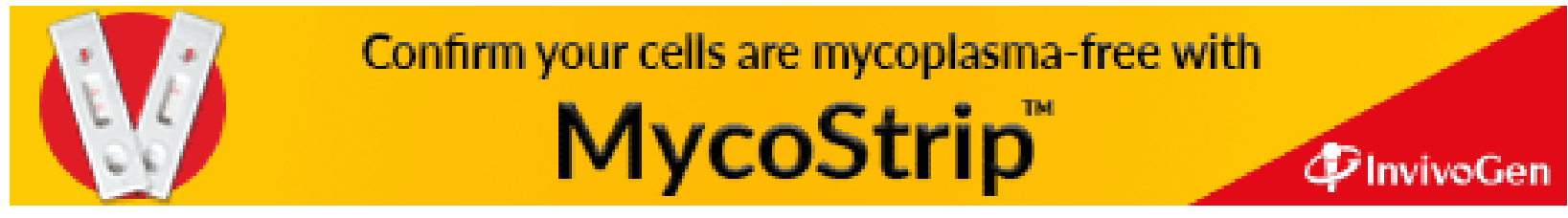

